\title{
The distribution of Blastocystis subtypes in isolates from Qatar
}

\author{
Marawan Abu-Madi ${ }^{*}$, Mahmoud Aly ${ }^{2,3}$, Jerzy M. Behnke ${ }^{4}$ C. Graham Clark ${ }^{5}$ and Hanan Balkhy ${ }^{2,3}$
}

\begin{abstract}
Background: Blastocystis is a common single-celled intestinal parasite of humans and other animals comprising at least 17 genetically distinct small subunit ribosomal RNA lineages (subtypes (STs)), nine of which have been found in humans. The geographic distribution of Blastocystis subtypes is variable, but the subtypes present in Qatar are at present unknown.
\end{abstract}

Methods: Stool samples were collected from randomly selected, apparently healthy subjects arriving in Qatar for the first time. Blastocystis subtypes were determined by sequencing of the small subunit rRNA gene (SSU rDNA) PCR products. Phylogenetic analyses were done using Maximum Composite Likelihood method.

Results: $71.1 \%$ of samples were positive for Blastocystis infection based on PCR-detection methodology compared to only $6.9 \%$ by microscopy. Prevalence of Blastocystis did not differ between the sexes nor between age classes. However, there was a regional difference in prevalence with subjects arriving from Africa showing the highest $(87.6 \%)$, those from Western Asia intermediate (68.6\%) and from Eastern Asia the lowest prevalence (67.6\%). Genetic analysis detected only three STs. ST3 was the most common (69.3\%) and ST2 was the rarest (3.5\%), while ST1 had a prevalence of $27.2 \%$. ST2 showed a regional variation, being absent from the 64 Western Asian Blastocystis-positive subjects. Both ST1 and ST3 showed significant differences in prevalence between the sexes.

Conclusions: This is the first report exploring the distribution of Blastocystis subtypes in our region. We recommend that stool screening via microscopy for the presence of Blastocystis should be abandoned since it is extremely insensitive. In future, the prevalence of Blastocystis infections should be based on PCR methodology and we predict that in the years ahead diagnostic PCR will become the tool of choice. More work is needed to identify the full range of Blastocystis subtypes that circulate in our region.

Keywords: Blastocystis, Real time-PCR, Subtype, Small subunit ribosomal DNA, Prevalence, Genotyping, Phylogenetic analysis, Qatar

\section{Background}

Blastocystis is a single-celled intestinal protist, taxonomically placed within the Stramenopiles, which colonizes an estimated 1,000,000,000 people globally and a variety of animal species [1]. Although Blastocystis is also detected in asymptomatic humans [2], some studies link this organism with intestinal and extra-intestinal disease $[3,4]$. To date, despite Blastocystis being the most frequently isolated protist from diarrheal patients in the developed world, a causal link has not yet been established conclusively [5]. Blastocystis is genetically diverse and

\footnotetext{
* Correspondence: abumadi@qu.edu.qa

${ }^{1}$ Department of Health Sciences, College of Arts and Science, Biomedical Research Center, Qatar University, P.O. Box 2713, Doha, Qatar

Full list of author information is available at the end of the article
}

based on small subunit rRNA gene analysis (SSU rDNA), at least 17 subtypes (STs) have been identified in humans, other mammals, and birds. Among these subtypes, ST1-ST4 collectively account for $90 \%$ of human carriage [6], while the ST5-ST9 account for the remaining $10 \%$. To date ST9 has only been isolated from humans [7]. To better understand the genetic diversity and determine the prevalence of Blastocystis subtypes around the world, further research is required [8, 9]. The introduction of molecular screening assays such as real-time polymerase chain reaction (RT-PCR) has demonstrated that the prevalence of Blastocystis is much higher than previously reported on the basis of detection by conventional microscopy, in both developed $[2,9,10]$ 
and developing [6] countries. Studying the genetic diversity of Blastocystis in different hosts, age groups and genders, and in different regions of the world, is essential to further our knowledge of the epidemiology and clinical relevance of this organism.

In the past decade, Qatar has seen a fast-paced transformation in the standards of living of its citizens. This tremendous growth has spawned building and modernization programs. There has been a large influx of migrant workers into Doha to complete very ambitious and large-scale construction projects. These workers mostly originate from states in the Middle-East, Asia and Africa, the latter two being regions where intestinal parasites are particularly common $[11,12]$. Economic growth has been accompanied by rapidly expanding domestic services in the city, which are also dependent on an immigrant labour force. Improvements in the infrastructure have not kept pace with this transformation, particularly the housing and sanitation available for workers. The immigration policy in Qatar applies a quota system to control the number of workers entering Qatar each year according to their country of origin. This creates a dynamic population of workers that changes from year to year and therefore requires continuous monitoring. On arrival in Qatar and before they can obtain work permits, all new immigrants are obliged to report to the Medical Commission for thorough medical inspections but fecal examinations of all immigrant workers seeking employment in the food industries and/ or as housemaids were abandoned following the introduction of compulsory treatment with albendazole, which is effective mainly against helminths and not most protozoa (although it does have activity against Giardia [13]). No specific treatment is given for protozoan infections, so in contrast to helminth infections these are not eradicated from infected immigrants on arrival in Qatar. Once a worker has been issued a work permit they are then not obliged to undergo further health inspections unless they work in the food industry, in which case annual reexamination is mandatory. As foreign workers form an integral part of the food industry in Qatar, it is imperative to determine their status as a potential reservoir and source of infection for enteric pathogens. The same is true for housemaids, who not only handle the food served to families, but also infants and children, who are generally more susceptible to infection than adults.

As prevention is far more effective in the long-term than cure, the identification of carriers and facilitation of the medical management of individuals who shed these pathogens can be improved through the application of sensitive screening methods, thereby minimizing the risk of infection spreading to other sectors of the community. In this paper we report on the implementation of a molecular screening assay (MSA) based on real-time PCR for the detection and subtyping of Blastocystis in samples collected from migrant workers newly arriving in Qatar, and we compare the detection rate with that achieved by conventional microscopy. We examine prevalence data for evidence of regional variation, while controlling for age and sex effects, and in a subset of the data we test whether the prevalence of the different STs of Blastocystis differs between regions of origin of the carriers or is influenced by host intrinsic factors, sex and age.

\section{Methods}

\section{Sample collection and DNA extraction}

We obtained stool samples from 608 randomly selected immigrant workers (including food handlers, construction workers, and housemaids), arriving for the first time in Qatar, and undergoing mandatory health checks at the Medical Commission during their application for work permits. Samples were collected in individually labelled, standard sterile disposable containers and were immediately stored on ice until processed for DNA extraction and microscopy.

Samples were aliquoted for DNA extraction alongside conventional stool examination by microscopy. Microscopic examination was carried out as described by Abu-Madi and others [14]. DNA was extracted using the QIAamp DNA stool minikit (Qiagen, Hilden, Germany) according to the manufacturer's instructions. Briefly, samples were weighed, homogenized in lysis buffer, and incubated at $95{ }^{\circ} \mathrm{C}$ for $5 \mathrm{~min}$ to ensure lysis of the targeted protozoa. After centrifugation, the DNA in the supernatant was purified using a silica column supplied with the kit. The quantity and quality of the DNA was determined using spectrophotometry (Nanodrop, ThermoScientific) and gel electrophoresis.

\section{Ethical approval}

Ethical approval for this project was obtained from the Medical Research Centre and the Research Committee at Hamad Medical Corporation, Qatar (Research protocol \# 11110/11).

\section{RT-PCR amplification and sequencing}

To detect the presence of Blastocystis in the DNA extracted from the samples described above, well-studied primers targeting the SSU rDNA region were used [10, $15,16]$. This region covers approximately $190 \mathrm{bp}$ that are highly specific for the SSU rDNAs of Blastocystis STs1-9, but can also distinguish each ST. This approach has been chosen over other possible approaches as the primer set used is of diagnostic quality yet at the same time it amplifies a product that will allow for subtyping once it is sequenced $[15,16]$. DNA extracted from samples that were positive for Blastocystis based on light microscopy was used as a positive control. The reaction mixture contained a final concentration of $0.3 \mu \mathrm{M}$ of the primer set and 
$0.125 \mu \mathrm{M}$ probe. Samples were processed using an ABI 7500 instrument. The reaction conditions were set as following: $95^{\circ} \mathrm{C}$ for $5 \mathrm{~min}$, and 50 cycles of denaturation at $95^{\circ} \mathrm{C}$ for $15 \mathrm{~s}$ followed by annealing and extension at $60{ }^{\circ} \mathrm{C}$ for $1 \mathrm{~min}$.

Purified PCR products were sequenced using a BigDye $^{\circ}$ Terminator v3.1 Cycle Sequencing Kit (Applied Biosystems ${ }^{\text {Th }}$ Austin, TX, USA). Thermal cycling was $96^{\circ} \mathrm{C}$ for $1 \mathrm{~min}, 40$ cycles of $\left(96{ }^{\circ} \mathrm{C}\right.$ for $10 \mathrm{~min}, 50{ }^{\circ} \mathrm{C}$ for $5 \mathrm{~s}$, $60{ }^{\circ} \mathrm{C}$ for $4 \mathrm{~min}$ ) using either Blastocystis Fwd S1, GGTCCGGTGAACACTTTGGATTT or Blastocystis RvsS2, CCTACGGAAACCTTGTTACGACTTCA. Products were purified using XTerminator ${ }^{\mathrm{rm}}$ and $\mathrm{SAM}^{\mathrm{sw}}$ solutions (Applied Biosystems ${ }^{\text {ta }}$ Foster City, CA, USA) and sequenced on a 3730xl DNA Analyzer (Applied Biosystems $^{\mathrm{Tw}}$, Hitachi, Tokyo, Japan).

\section{Genotyping analysis}

To identify Blastocystis subtypes, both strands of the amplicons were sequenced and were compared with all the SSU rRNA gene sequences available from the $\mathrm{Na}$ tional Centre for Biotechnology Information (NCBI) database using the BLAST program. SSU rDNA subtypes were identified by determining the closest similarity match against all known Blastocystis STs. All of our sequences have been deposited in GenBank under Accession numbers KM438204-30.

\section{Statistical analysis}

Prevalence data are presented as percentages with $95 \%$ confidence limits ( $95 \% \mathrm{CL}$ ), calculated with bespoke software based on the tables of Rohlf and Sokal [17]. Analysis comprised three stages. In the first we compared detection of Blastocystis using the PCR method with conventional detection by routine microscopy.

In the second step, we analyzed the factors affecting prevalence of Blastocystis, based on detection of infected subjects by PCR. For this analysis we used maximum likelihood techniques based on log linear analysis of contingency tables in the software package IBM SPSS Statistics Version 21 (IBM Corporation). Initially, full factorial models were fitted, incorporating as factors region of origin of subjects (REGION - 3 levels), sex of subjects (SEX, 2 levels, male and female), and age of subjects (AGE, 3 levels). The subjects $(n=608)$ originated from 18 countries, but for statistical analysis were assigned to three regions. Region 1, Africa comprised subjects from Cameroon ( $n=1)$, Egypt (2), Eritrea (3), Ethiopia (34), Ghana (3), Kenya (27), Nigeria (15), Sudan (2), Chad (1) and Uganda (1). Region 2, Eastern Asia comprised subjects from Indonesia (119), Philippines (65), Thailand (2) and Vietnam (2). Region 3, Western Asia, comprised subjects from Bangladesh (71), India (117), Nepal (62) and Sri Lanka (81). Subjects were allocated to three age classes (age class $1=18-29$ years old, age class $2=30-39$ years old and age class $3=40-58$ years old). The presence / absence of Blastocystis (INFECTION) was fitted as a binary factor. These explanatory factors were fitted initially to all models that were evaluated. For each level of analysis in turn, beginning with the most complex model, involving all possible main effects and interactions, those combinations that did not contribute significantly to explaining variation in the data were eliminated in a stepwise fashion beginning with the highest level interaction (backward selection procedure). A minimum sufficient model was then obtained, for which the likelihood ratio of $\chi 2$ was not significant, indicating that the model was sufficient to explain the data. The importance of each term (i.e. interactions involving infection) in the final model was assessed by the probability that its exclusion would alter the model significantly and these values relating to interactions that included INFECTION are given in the text.

In stage three, analysis of the factors affecting the prevalence of genotyped Blastocystis isolates was conducted on the smaller data-set of sequenced isolates. The subtype identification of a subset of 132 randomly selected isolates was achieved by sequencing the SSU rDNA. Some isolates $(n=18)$ were problematic and not available for analysis because they could not be subtyped successfully or were associated with missing data. However, 114 isolates were completely subtyped and analysed for the current study. The successfully subtyped isolates originated from donors ranging in age from 20 years to a maximum of 56 years, with an average of $31.9 \pm 0.75$ (median of 30.0) years. Fifty three subjects were males (mean age $=31.2 \pm 1.13$ and 61 females (mean age $=$ $32.5 \pm 0.99$ ), and there was no significant difference between the ages of the two sexes $(z=1.055, P=0.29)$. The approach adopted for analysis was similar to that in stage two of the analysis. Again subjects were allocated to the same three regions and age classes. Three models were fitted, one each for Blastocystis ST1, ST2 and ST3, and in each case with AGE, SEX and REGION as explanatory factors and presence/absence of the subtype (INFECTION) as a binary factor. Reduction to minimum sufficient models followed the same procedure as that explained above in stage two of the analysis.

Throughout our cut-off for statistical significance was $P=0.05$.

\section{Results}

\section{Prevalence of Blastocystis based on PCR method of} detection

Of the 608 samples tested by PCR for the presence of Blastocystis, 432 were positive and 176 negative giving an overall prevalence of $71.1 \%$. In contrast, microscopy only identified 42 positives ( $6.9 \%$ prevalence). Thirty seven samples were identified as positive and 171 as 
negative by both methods. However, five samples were recorded as positive by microscopy but not detected as such by PCR.

\section{Factors affecting the prevalence of Blastocystis based on detection by PCR}

Analysis of the prevalence of Blastocystis based on PCR revealed that neither host sex nor age affected prevalence significantly (Table 1 ). However, there was a highly significant effect of the region from which subjects originated (REGION x INFECTION, $\chi_{2}^{2}=16.1, p<0.001$ ). Infections with Blastocystis were most common among subjects from Africa and least common among those from Eastern Asia, and the difference in prevalence between these regions was $20.0 \%$.

\section{Subtype analysis and prevalence}

Subtype analysis revealed that only three subtypes were present in the samples analyzed. ST1 was present in 31 samples (27.2\%), ST2 was found in four hosts (3.5\%), one from Nigeria and three from Indonesia, while 79 (69.3\%) samples had ST3. Interestingly, ST3 was found in samples isolated from subjects of all geographical regions included in the current study i.e. Africa, East and West Asia. Table 2 shows the occurrence of subtypes and strains among the 114 subjects by country of origin. When the data were subdivided by the 12 countries of origin of the subjects, some subsets became very small, and indeed there was only one subject each from Cameroon, Chad and Eritrea.

\section{Factors affecting the prevalence of subtypes of} Blastocystis in subsets of the subjects in the study In order to enable statistical analysis, the subjects were allocated to three geographical regions (Tables 2 and 3). There was no regional effect on ST1 and ST3, but with age and host sex taken into account there was a

Table 1 Factors affecting prevalence of Blastocystis, based on detection by PCR

\begin{tabular}{llcl}
\hline Factor & Number & Prevalence (\%) & $95 \% \mathrm{CL}$ \\
\hline Host sex & & & \\
$\quad$ Males & 280 & 70.7 & $65.85-75.17$ \\
$\quad$ Females & 328 & 71.3 & $66.10-76.10$ \\
Host age & & & \\
Age class 1 (18-29) & 286 & 73.1 & $68.25-77.42$ \\
Age class 2 (30-39) & 233 & 70.0 & $65.49-74.10$ \\
Age class 3 (40-58) & 89 & 67.4 & $54.32-78.65$ \\
Region of origin & & & \\
Africa & 89 & 87.6 & $76.54-94.08$ \\
Eastern Asia & 188 & 67.6 & $57.95-75.86$ \\
$\quad$ Western Asia & 331 & 68.6 & $63.23-73.49$ \\
\hline
\end{tabular}

Table 2 The distribution of subtypes of Blastocystis among the 114 subjects by their countries of origin

\begin{tabular}{lllll}
\hline Nationality & $n$ & ST1 & ST2 & ST3 \\
\hline Africa & 19 & & & \\
Cameroon & 1 & 1 & 0 & 0 \\
Chad & 1 & 1 & 0 & 0 \\
Eritrea & 1 & 1 & 0 & 0 \\
Ethiopia & 12 & 7 & 0 & 5 \\
Kenya & 2 & 0 & 0 & 2 \\
Nigeria & 2 & 0 & 1 & 1 \\
East Asian & 31 & & & \\
Indonesia & 19 & 6 & 3 & 10 \\
Philippines & 12 & 4 & 0 & 8 \\
West Asia & 64 & & & \\
Bangladesh & 12 & 3 & 0 & 9 \\
India & 20 & 3 & 0 & 17 \\
Nepal & 16 & 1 & 0 & 15 \\
Sri Lanka & 16 & 4 & 0 & 12 \\
& 114 & 31 & 4 & 79 \\
\hline
\end{tabular}

significant effect of region of origin on ST2 (REGION x INFECTION, $\chi_{2}^{2}=7.1, P=0.029$ ), despite the low prevalence of this ST among the 114 samples. This subtype was not detected among the $64 \mathrm{~W}$. Asian subjects (Table 2). Table 3 shows that ST3 appeared to be more common among W. Asian and ST1 among African subjects, but with host sex and age taken into account these differences in prevalence did not reach statistical significance (effect of REGION on ST1, $\chi_{2}^{2}=5.1, P=0.078$ and on ST3, $\chi_{2}^{2}=5.9, P=0.052$ ).

With region of origin and age taken into account, there were significant effects of host sex on the prevalence of ST1 (Table 4; SEX x INFECTION, $\chi_{1}^{2}=13.4, p$ $<0.001$ ) and ST3 (Table 4; SEX $\times$ INFECTION, $\chi_{1}^{2}=15.1$, $p<0.001)$. In the case of ST1 the bias was strongly in favour of female subjects with prevalence of this subtype being 3.6-fold more common among females. ST3, showed a bias in the opposite direction with prevalence among male subject being 1.6-fold higher compared with females.

With region of origin and host sex taken into account, there were no significant effects of host age, although as

Table 3 Prevalence of subtypes of Blastocystis among the three regions of origin of the subjects in the study

\begin{tabular}{lllll}
\hline Region & $n$ & \multicolumn{3}{l}{ Subtype prevalence as a percentage $(95 \% \mathrm{CL})$} \\
\cline { 3 - 5 } & & ST1 & ST2 & ST3 \\
\hline Africa & 18 & $52.6(31.18-74.29)$ & $5.3(0.27-25.70)$ & $42.1(22.19-65.51)$ \\
East Asia & 28 & $32.3(20.18-46.45)$ & $9.7(3.77-21.51)$ & $58.1(43.81-71.40)$ \\
West Asia & 64 & $17.2(10.19-27.20)$ & $0(0-4.80)$ & $82.8(72.80-89.81)$ \\
\hline
\end{tabular}


Table 4 The influence of host sex and age on the prevalence of ST1 and ST3

\begin{tabular}{|c|c|c|c|c|}
\hline \multirow[t]{2}{*}{ Subset } & \multicolumn{2}{|l|}{ ST1 } & \multicolumn{2}{|l|}{ ST3 } \\
\hline & $\%$ & $95 \% \mathrm{CL}$ & $\%$ & $95 \% \mathrm{CL}$ \\
\hline Males & 11.3 & $6.12-19.43$ & 86.8 & $78.52-92.57$ \\
\hline Females & 41.0 & $30.62-51.87$ & 54.1 & $43.19-64.47$ \\
\hline Age class 1 (18-29) & 30.0 & $18.80-43.96$ & 66.0 & $52.02-78.23$ \\
\hline Age class 2 (30-39) & 27.3 & $14.46-44.25$ & 68.2 & $51.16-82.03$ \\
\hline Age class $3(40-58)$ & 20.0 & $7.14-42.35$ & 80.0 & $57.65-92.86$ \\
\hline
\end{tabular}

can be seen from Table 4, there appeared to be a steady decline in the prevalence of ST1 with increasing age and an increase with age in the case of ST3.

\section{Discussion}

The results of our study show clearly that there is an enormous difference in the detection of Blastocystis in stool samples by microscopy vs PCR methodology. Based on PCR methodology we concluded that $71.1 \%$ of the samples contained Blastocystis DNA, whilst conventional microscopy detected only 42 positive samples, giving a prevalence of just $6.9 \%$. Higher detection rates of intestinal protozoa, including Blastocystis, by PCR compared with conventional microscopy have also been recently reported in asymptomatic individuals from Brazil [18], where microscopy detected 10 infected subjects out of 126 (prevalence $=7.9 \%$ ) whereas PCR detected 43 infections in the same subjects (prevalence $=34.1 \%$ ). This difference in sensitivity of the two assays raises questions about the utility of continuing to employ conventional microscopy, when this method has such a high failure rate in detecting the presence of this parasite. In this study, as in our earlier work from Qatar, the stool samples were all screened by highly trained medical laboratory technicians and it is unlikely that any further improvement is possible in the accuracy of detection by microscopy. On this basis, we can confidently conclude that earlier estimates of the prevalence of Blastocystis in Qatar, which were all entirely based on microscopy [14], were very heavily underestimated. Clearly, diagnostic microscopy of Blastocystis infections is generally less sensitive than PCR-based methodology, resulting in marked underestimation of the true prevalence of Blastocystis.

Our data showed that there was no difference in prevalence between males and females both having similarly high values for prevalence, although others have found significant differences in prevalence between the sexes. Higher prevalence of Blastocystis in male compared with female subjects has been observed, for example, in Libya [19] and in China [20]. Participation in outdoor activities by the adult males, with the associated higher risk of contamination by the faecalorally transmitted cysts of the parasite, has been proposed as the underlying explanation. However, there are examples also of bias in the opposite direction with higher prevalence among female subjects, as for example in Spain [21].

There was no difference in prevalence between the age classes in our data, although there was a consistent, if marginal, drift downwards with increasing age, which was not statistically significant. Some earlier studies concur with our results in failing to find any age effects on the prevalence of Blastocystis. For example, no significant associations were found between infections with any of the observed Blastocystis STs and age classes in Libya [22]. However, other studies have found significantly higher infection rates in adults compared with children, with the highest prevalence rate among asymptomatic young adults aged between 18 and 30 years [23]. Trends in the opposite direction, with a higher prevalence rate in children compared to adults, have also been reported, as for example in the Philippines [24]. In support of the latter, a recent study in Thailand has also reported a significant reduction in the Blastocystis infection with increasing age, the prevalence rate peaking in the younger children in the study [25]. These contrasting findings suggest that the distribution of Blastocystis infection among host populations shows spatial heterogeneity with respect to host age and sex, and these inconsistencies are most likely attributable to local factors such as the environmental conditions that influence locally the extent of, and the efficiency of, the faecal-oral route of transmission among host sectors of varying age, and between the two sexes [22].

The most prominent source of variation in prevalence of Blastocystis that we detected was in the region of origin of the subjects, with immigrants from Africa showing the highest prevalence and those from Eastern Asia, comprising Indonesia and the Philippines, the lowest. The prevalence of Blastocystis infection is known to vary from country to country, as well as within countries [6]. However, it has been reported that a higher prevalence occurs in developing countries than developed countries [26-28]. Blastocystis is transmitted through the faecaloral route and among the most obvious risk factors are poor personal and community hygiene, culture, and lifestyle of a population. All of these vary from country to country, partially depending on the economic status and standard of living, but also on the geographical location and climatic factors [29].

Because we used a PCR-based methodology for detection and successfully sequenced the PCR products, we were also able to distinguish between different STs of Blastocystis. Our results revealed some interesting trends with respect to the influence of the region of origin on the prevalence of STs but the only ST that was shown statistically to vary in relation to where the carrier originated 
from was ST2, which was completely absent in the 64 subjects from W. Asia. It should be noted that only four samples in total were classified as this ST, and therefore we have to reserve judgement as to whether the conclusion of a regional influence on the prevalence of this ST is robust. Clearly further work must include a considerably larger sample size, to consolidate or refute this finding.

The high prevalence of ST3 in our study population, especially among immigrants from Western Asia, is consistent with other reports in the literature, as for example in Thailand [30], Egypt [31], Singapore [26], Turkey [32, 33], Germany [34], France [35], Malaysia [36], and Lebanon [37]. In a recent study of the distribution of Blastocystis subtypes in three African countries (Libya, Nigeria and Liberia) by Alfellani et al. [6], four subtypes were detected in the Libyan population with ST1 $(50.0 \%, 19 / 38)$ showing the highest prevalence, followed by ST3 $(39.5 \%, 15 / 38)$, ST2 $(7.9 \%, 3 / 38)$ and ST7 $(2.6 \%, 1 / 38)$. Other studies have also identified ST1 as dominant among the examined outpatients [19].

Perhaps the most interesting finding from our study, was the marked sex-difference in the prevalence of ST1 and ST3, with ST1 being far more common among female subjects and subtype 3 more common among infected males. Our finding of female bias with ST1 agrees with a study in Libya where Blastocystis ST1 infection was also significantly associated with females but was linked also to a low educational level [19]. Nevertheless, female sex-bias has not been detected in all studies. No significant associations between sex and subtypes of Blastocystis were found in Turkey $[33,34,38]$. The reasons for these marked differences in the relative proportions of subtypes between the sexes in affected populations are not currently understood, but they most likely relate to differences between the sexes in cultural/traditional patterns of behaviour and hence to different exposure rates to the sources of transmission [39].

\section{Conclusions}

On the basis of our results, we recommend that stool screening via microscopy for the presence of Blastocystis should be largely abandoned since it is extremely insensitive even in the hands of the most experienced technicians. In future, the detection of Blastocystis infections should be based on PCR methodology and we predict that in the years ahead diagnostic PCR will become the tool of choice [40]. Our results have raised a number of interesting issues that will be addressed in future work, notably the underlying reasons for the regional trends in the prevalence of the three STs of Blastocystis that we detected in our study population, and for the sex bias in the prevalence of ST1 and ST3.

\section{Competing interests}

The authors declare that they have no competing interests.

\section{Authors' contributions}

MAAM and JMB conceived the study, collected and analyzed the data, and wrote the manuscript. CGC analyzed and interpreted the results and wrote the manuscript. MA and HB performed the genotyping and DNA sequencing. All authors read and approved the final manuscript.

\section{Acknowledgements}

We would like to thank the Biomedical Research Centre at Qatar University for providing facilities for this work. This publication was made possible by a NPRP grant number NPRP4-1283-3-327 from the Qatar National Research Fund, Qatar Foundation and HMC grant \# 11110/11. The statements made herein are solely the responsibility of the authors. We would like to thank Haneen Al-Bardaweel and Aarti Sharma for their help in processing the samples. All authors read and approved the final version of the manuscript.

\section{Author details}

${ }^{1}$ Department of Health Sciences, College of Arts and Science, Biomedical Research Center, Qatar University, P.O. Box 2713, Doha, Qatar. ${ }^{2}$ King Abdullah International Medical Research Center, National Guard Health Affairs, Mail Code: 2216, P.O. Box 22490, Riyadh 11426, KSA. ${ }^{3}$ King Saud bin Abdulaziz University for Health Sciences, Riyadh, Saudi Arabia. ${ }^{4}$ School of Life Sciences, University of Nottingham, University Park, Nottingham, United KingdomNG7 2RD. ${ }^{5}$ Faculty of Infectious and Tropical Diseases, London School of Hygiene and Tropical Medicine, Keppel Street, WC1E 7HT London, United Kingdom.

Received: 5 July 2015 Accepted: 3 September 2015

Published online: 17 September 2015

\section{References}

1. Scanlan PD, Stensvold CR. Blastocystis: getting to grips with our guileful guest. Trends Parasitol. 2013;29:523-9.

2. Scanlan PD, Marchesi JR. Micro-eukaryotic diversity of the human distal gut microbiota: qualitative assessment using culture-dependent and -independent analysis of faeces. ISME J. 2008;2:1183-93.

3. Jimenez-Gonzalez DE, Martinez-Flores WA, Reyes-Gordillo J, Ramirez-Miranda ME, Arroyo-Escalante S, Romero-Valdovinos M, et al. Blastocystis infection is associated with irritable bowel syndrome in a Mexican patient population. Parasitol Res. 2012;110:1269-75.

4. Zuel-Fakkar NM, Abdel Hameed DM, Hassanin OM. Study of Blastocystis hominis isolates in urticaria: a case-control study. Clin Exp Dermatol. 2011;36:908-10.

5. Fletcher SM, Stark D, Harkness J, Ellis J. Enteric protozoa in the developed world: a public health perspective. Clin Microbiol Rev. 2012;25:420-49.

6. Alfellani MA, Stensvold CR, Vidal-Lapiedra A, Onuoha ES, Fagbenro-Beyioku AF, Clark CG. Variable geographic distribution of Blastocystis subtypes and its potential implications. Acta Trop. 2013;126:11-8. doi:10.1016/ j.actatropica.2012.12.011. PubMed: 23290980

7. Ramírez JD, Sánchez LV, Bautista DC, Corredor AF, Flórez AC, Stensvold CR. Blastocystis subtypes detected in humans and animals from Colombia. Infect Genet Evol. 2014;22:223-8.

8. Stensvold CR, Nielsen HV, Mølbak K, Smith HV. Pursuing the clinical significance of Blastocystis-diagnostic limitations. Trends Parasitol. 2009;25:23-9.

9. Stensvold CR, Christiansen DB, Olsen KE, Nielsen HV. Blastocystis sp subtype 4 is common in Danish Blastocystis-positive patients presenting with acute diarrhea. Am J Trop Med Hyg. 2011;84:883-5.

10. Forsell J, Granlund M, Stensvold CR, Clark CG, Evengård B. Subtype analysis of Blastocystis isolates in Swedish patients. Eur J Clin Microbiol Infect Dis. 2012;31:1689-96.

11. Dib HH, Lu SQ, Wen SF. Prevalence of Giardia lamblia with or without diarrhea in South East, South East Asia and the Far East. Parasitol Res. 2008;103:239-51.

12. Nkrumah B, Nguah SB. Giardia lamblia: a major parasitic cause of childhood diarrhoea in patients attending a district hospital in Ghana. Parasit Vectors. 2011;4:163. doi:10.1186/1756-3305-4-163.

13. Reynoldson JA, Behnke JM, Gracey M, Horton RJ, Spargo R, Hopkins RM, et al. Efficacy of albendazole against Giardia and hookworm in a remote Aboriginal community in the north of Western Australia. Acta Trop. 1998;71:27-44 
14. Abu-Madi MA, Behnke JM, Doiphode SH. Intestinal parasitic infections among long-term residents and settled immigrants in Qatar in the period 2005 to 2011. Am J Trop Med Hyg. 2013;88:1185-95. doi:10.4269/ajtmh.13-0006.

15. Stensvold CR, Ahmed UN, Andersen LO, Nielsen HV. Development and evaluation of a genus-specific, probe-based, internal-process-controlled real-time PCR assay for sensitive and specific detection of Blastocystis spp. J Clin Microbiol. 2012;50:1847-51.

16. Stensvold CR, Alfellani MA, Nørskov-Lauritsen S, Prip K, Victory EL, Maddox C, et al. Subtype distribution of Blastocystis isolates from synanthropic and zoo animals and identification of a new subtype. Int J Parasitol. 2009;39:473-9.

17. Rohlf FJ, Sokal RR. Statistical Tables. San Francisco: Freeman W.H. \& Company; 1995

18. David EB, Guimarães $S$, de Oliveira AP, de Oliveira-Sequeira TCG, Bittencourt GN, Nardi ARM, et al. Molecular characterization of intestinal protozoa in two poor communities in the State of São Paulo Brazil. Parasit Vectors. 2015:8:103.

19. Abdulsalam AM, Ithoi I, Al-Mekhlafi HM, Al-Mekhlafi AM, Ahmed A, Surin J. Subtype distribution of Blastocystis isolates in Sebha, Libya. PLoS One. 2013;8:e84372. doi:10.1371/journal.pone.0084372.

20. Wang KX, Li CP, Wang J, Cui YB. Epidemiological survey of Blastocystis hominis in Huainan City, Anhui Province, China. World J Gastroenterol. 2002;8:928-32.

21. Martin-Sanchez A, Canut-Blasco A, Rodriguez-Hernandez J, Montes-Martinez I, Garcia-Rodriguez J. Epidemiology and clinical significance of Blastocystis hominis in different population groups in Salamanca (Spain). Eur J Epidemiol. 1992;8:553-9.

22. Abdulsalam AM, Ithoi I, Al-Mekhlafi HM, Khan A, Ahmed A, Surin J, et al. Prevalence, predictors and clinical significance of Blastocystis sp. in Sebha, Libya. Parasit Vectors. 2013;6:86. http://www.biomedcentral.com/content/ pdf/1756-3305-6-86.pdf.

23. Yaicharoen R, Sripochang S, Sermsart B, Pidetcha P. Prevalence of Blastocystis hominis infection in asymptomatic individuals from Bangkok, Thailand. Southeast Asian J Trop Med Public Health. 2005;36:17-20.

24. Baldo ET, Belizario WY, De Leon WU, Kong H-H, Chung D-I. Infection status of intestinal parasites in children living in residential institutions in Metro Manila, the Philippines. Korean J Parasitol. 2004;42:67-70.

25. Pipatsatitpong D, Rangsin R, Leelayoova S, Naaglor T, Mungthin M Incidence and risk factors of Blastocystis infection in an orphanage in Bangkok, Thailand. arasit Vectors. 2012;14:37.

26. Wong $\mathrm{KH}, \mathrm{Ng} \mathrm{GC}$, Lin RT, Yoshikawa $\mathrm{H}$, Taylor MB, Tan KS. Predominance of subtype 3 among Blastocystis isolates from a major hospital in Singapore. Parasitol Res. 2008;102:663-70.

27. Hirata T, Nakamura H, Kinjo N, Hokama A, Kinjo F, Yamane N, et al. Prevalence of Blastocystis hominis and Strongyloides stercoralis infection in Okinawa, Japan. Parasitol Res. 2007;101:1717-9.

28. Horiki N, Maruyama M, Fujita Y, Yonekura T, Minato S, Keneda Y. Epidemiologic survey of Blastocystis hominis infection in Japan. Am J Trop Med Hyg. 1997;56:370-4.

29. Popruk S, Udonsom R, Koompapong K, Mahittikorn A, Kusolsuk T, Ruangsittichai J, et al. Subtype distribution of Blastocystis in Thai-Myanmar border, Thailand. Korean J Parasitol. 2015;53:13-9. doi:10.3347/ kjp.2015.53.1.13.

30. Yoshikawa H, Wu Z, Kimata I, Iseki M, Ali IK, Hossain MB, et al. Polymerase chain reaction-based genotype classification among human Blastocystis hominis populations isolated from different countries. Parasitol Res. 2004:93:22-9.

31. Hussein EM, Hussein AM, Eida MM, Atwa MM. Pathophysiological variability of different genotypes of human Blastocystis hominis Egyptian isolates in experimentally infected rats. Parasitol Res. 2008;102:853-60.

32. Böhm-Gloning B, Knobloch J, Walderich B. Five subgroups of Blastocystis hominis isolates from symptomatic and asymptomatic patients revealed by restriction site analysis of PCR amplified 16S-like rDNA. Trop Med Int Health. 1997:2:771-8.

33. Özyurt M, Kurt Ö, Mølbak K, Nielsen HV, Haznedaroglu T, Stensvold CR. Molecular epidemiology of Blastocystis infections in Turkey. Parasitol Int. 2008;57:300-6.

34. Dogruman-Al F, Yoshikawa H, Kustimur S, Balaban N. PCR-based subtyping of Blastocystis isolates from symptomatic and asymptomatic individuals in a major hospital in Ankara, Turkey. Parasitol Res. 2009;106:263-8.
35. Souppart L, Sanciu G, Cian A, Wawrzyniak I, Delbac F, Capron M. Molecular epidemiology of human Blastocystis isolates in France. Parasitol Res. 2009;105:413-21

36. Ragavan ND, Govind SK, Chye TT, Mahadeva S. Phenotypic variation in Blastocystis sp. ST3. Parasit Vectors. 2014;7:404.

37. El Safadi D, Meloni D, Poirier P, Osman M, Cian A, Gaayeb L, et al. Molecular epidemiology of Blastocystis in Lebanon and correlation between Subtype 1 and gastrointestinal symptoms. Am J Trop Med Hyg. 2013;88:1203-6. doi:10.4269/ajtmh.12-0777.

38. Dogruman-Al F, Dagci H, Yoshikawa H, Kurt K, Demirel M. A possible link between subtype 2 and asymptomatic infections of Blastocystis hominis. Parasitol Res. 2008;103:685-9. doi:10.1007/s00436-008-1031-3. PubMed: 18523804.

39. Li LH, Zhou XN, Du ZW, Wang XZ, Wang LB, Jiang JY, et al. Molecular epidemiology of human Blastocystis in a village in Yunnan province, China. Parasitol Int. 2007;56:281-6. doi:10.1016/j.parint.2007.06.001. PubMed: 17627869.

40. Clark CG, van der Giezen M, Alfellani MA, Stensvold CR. Recent developments in Blastocystis research. Adv Parasitol. 2013:82:1-32. doi:10.1016/B978-0-12-407706-5.00001-0.

\section{Submit your next manuscript to BioMed Central and take full advantage of:}

- Convenient online submission

- Thorough peer review

- No space constraints or color figure charges

- Immediate publication on acceptance

- Inclusion in PubMed, CAS, Scopus and Google Scholar

- Research which is freely available for redistribution 